\title{
The potential influence of machine learning and data science on the future of economics: Overview of highly-cited research
}

\author{
Advait Deshpande \\ Current version May 2020
}

\begin{abstract}
This working paper provides an overview of the potential influence of machine learning and data science on economics as a field. The findings presented are drawn from highly cited research which was identified based on Google Scholar searches. For each of the articles reviewed, this working paper covers what is likely to change and what is likely to remain unchanged in economics due to the emergence and increasing influence of machine learning and data science methods. The findings presented are initial and exploratory. Further analysis of the literature would be necessary to develop a more definitive understanding of the likely changes to economics.
\end{abstract}

\section{Introduction}

The aim of this working paper is to explore the potential influence of machine learning and data science on the future of economics. ${ }^{1}$ It does so by synthesising the arguments presented in five highly cited research articles examining the likely changes in the field of economics due to the increasing influence of machine learning in collecting and analysing data. These arguments are considered in the context of the increased use of computational methods in statistics, applied economic analysis (including econometrics), and the emergence of data science as a field of expertise on its own.

The next section discusses the approach taken to selecting the articles to identify what is likely to change and what is likely to remain the same as the use of machine learning and data science approaches becomes more common place in the field of economics.

\section{Approach to identifying relevant literature}

In order to identify the most relevant, highly cited articles, literature searches were conducted on Google Scholar with the following search string: 'impact+of+machine+learning+data science+in+economics'. ${ }^{2}$ Given the rapidly changing nature of the developments in machine learning and data science, only articles since 2015 onwards were considered. The search results were sorted for relevance and number of citations. Amongst the literature thus identified, only articles discussing either economics or econometrics at a broad-level were considered. Articles

\footnotetext{
${ }^{1}$ The views and opinions expressed in this article are those of the author and do not necessarily reflect the policy or position of any agency or organisation the author works for or has worked for in the past. The copyright for this work belongs to the author. This work is licensed under the Creative Commons Attribution-NonCommercial-NoDerivatives 4.0 International (CC BY-NC-ND 4.0) licence.

${ }^{2}$ Searches conducted in May 2020.
} 
discussing machine learning and data science in relation to specific sub-fields of economics (for example, Kou et al. 2019, and Hsu et al. 2016) were excluded. Similarly articles covering the impact of machine learning and data science as part of a 'future of work'-themed discussion (for example, Ernst, Merola, and Samaan 2019, Agrawal, Gans, and Goldfarb 2018a, 2018b) were also not considered. The exclusions were aimed at ensuring that a high-level perspective on potential changes to economics as a field could be collected as part of the findings. In the end, the following articles were considered: Mullainathan and Spiess (2017), Athey (2018), Athey and Imbens (2019), Athey (2015), and Athey and Luca (2019). ${ }^{3}$

The next section synthesises the findings reported in the above-mentioned five articles to understand the likely changes to economics as a field. Given the highly selective approach to identifying the articles, the following discussion is intended to be exploratory rather than presenting any definitive assessments. Further in-depth assessment of the available literature would be necessary to analyse how developments in machine learning and data science are likely to influence economics in the future.

\section{Findings}

In this section the findings from each of the five highly cited articles selected are discussed to understand the key themes explored in each articles, potential changes to economics due to the emergence of machine learning and data science, aspects of economics that are likely to be remain unchanged despite the increasing influence of machine learning and data science, and any other relevant observations. A summary of findings drawing on each of the five articles is presented at the end.

For the purposes of the following discussion, machine learning is defined as a field that develops algorithms designed to be applied to datasets, with the main areas of focus being prediction (regression), classification, and clustering or grouping tasks (Athey 2018). Although for the sake of simplicity no distinction is made between supervised and unsupervised machine learning approaches, the articles reviewed mainly appeared to discuss supervised machine learning. ${ }^{4}$ Data science is defined as a cross-disciplinary area of expertise drawing on knowledge of mathematics and statistics, software programming skills, and substantive domain expertise about the area of analysis or investigation (VanderPlas 2017).

\subsection{Findings from Mullainathan and Spiess (2017)}

\subsubsection{Key themes explored}

Mullainathan and Spiess (2017) discuss the ways in which machine learning could be adopted and adapted as part of an existing set of methods and approaches in econometrics. According to

\footnotetext{
${ }^{3}$ Articles listed in descending order based on the number of citations identified in Google Scholar at the time of the original searches.

${ }^{4}$ Unsupervised machine learning refers to machine learning approaches in which the input data is not labelled or categorised prior to the analysis. In supervised machine learning, a set of inputs (which are labelled or categorised) are used to predict outcomes. See Athey (2018) for a more detailed discussion.
} 
the authors, the key contribution of machine learning to econometrics is likely to be due to its ability incorporate data that is out of sample as part of complex systems. They aim to identify specific strengths of machine learning that could inform the way economists work and think about problem solving.

\subsubsection{Likely changes in economics}

According to Mullainathan and Spiess (2017), machine learning and data science are likely to find applications in economics when new kinds of (possibly unstructured) data need to be used for analysing, solving traditional economic and policy problems. In particular, the authors suggest that the use of machine learning is likely to be increasingly found in dealing with image data, language information, and unconventional data (e.g. satellite data) which does not fit standard estimation methods (including as part of regression models). The use of machine learning and data science methods is thus likely to not only increase the scope of economic work due to inclusion of new data but also aide the investigation of new questions.

\subsubsection{Aspects of economics that are likely to remain unchanged}

Mullainathan and Spiess (2017) argue that reliable estimate of predictive performance remains and will remain a non-negotiable requirement for economic modelling. At present, machine learning and data science approaches, although these are highly flexible, do not appear to provide robust methods of identifying standard errors. In consequence, machine learning and data science predictors even when they use linear functions, may not be perceived as providing reliable estimates when compared against traditional econometric models.

\subsection{Findings from Athey (2018)}

\subsubsection{Key themes explored}

Athey (2018) discusses the different ways in which machine learning is likely to contribute to and change economics as a field. In particular Athey (2018) explores the difference in thinking between computer science and economics as fields and the ways in which machine learning algorithms would need to be modified to fit the norms of economics in terms of robustness of data and probability of error.

\subsubsection{Likely changes in economics}

According to Athey (2018), one of the main changes that machine learning is likely to introduce to economics is the way in which machine learning algorithms estimate and compare alternative models. Athey (2018) highlights that in economics (in principle if not in practice) the researcher identifies the model once and then carries out estimations. The data driven nature of machine learning algorithms is likely to lead to changes to economists' role in design and implementation of policies. Additionally the use of machine learning is likely to result in collection of large datasets on individual behaviours. Athey (2018) suggests that this could mean new research questions relying on different measurement approaches to economic outcomes. New ways to develop predictive and inference models that are less susceptible to manipulation and better 
protected against poor data fit are also likely according to the author. According to Athey (2018), the emergence of machine learning (and artificial intelligence) is likely to result in new policy problems to study.

\subsubsection{Aspects of economics that are likely to remain unchanged}

Athey (2018) suggests that despite the introduction of machine learning, the theories that underpin the identification of causal effects in economics are unlikely to change. According to Athey (2018) this means the automation offered by machine learning algorithms does not change the need to ensure that the economics analysis should provide the accurate empirical assessments. The scale of automation offered by machine learning and data science approaches is also unlikely to change key practices in economics such as testing validity of the model based on outcomes predicted, or assessing whether causal effects identified are appropriate for the data used (Athey 2018).

\subsection{Findings from Athey and Imbens (2019)}

\subsubsection{Key themes explored}

Athey and Imbens (2019) explore the reasons for which adoption of machine learning methods has been slower in economics than statistical analysis. In particular, the instances in which offthe-shelf uses of machine learning algorithms are not useful and thus need to be applied in a way specific to the problems are considered. The authors aim to identify machine learning and data science based tools that they think should become part of an econometrics graduate course.

\subsubsection{Likely changes in economics}

Athey and Imbens (2019) highlight that economics literature and machine learning literature have very different approaches to problem solving. They suggest that unlike economics literature machine learning literature does not necessarily focus on causal effects or configuration of data to develop specific insights. The main adaptations in economics due to the emergence of machine learning are thus likely to be in the form of increased use of machine learning algorithms in specific settings to understand the potential outcomes. For example, the author suggest potential uses in relation to problems where formal results are about guarantees of error rates, or where problems rely on specific types of regression techniques that are less concerned with problems that draw on traditional economic theory.

\subsubsection{Aspects of economics that are likely to remain unchanged}

Athey and Imbens (2019) discuss how the requirements of economic journals in terms of use of methods do not naturally fit the approaches taken by machine learning literature. This suggests that in order to address problems economists are interested in, machine learning methods will need careful adaptation to deliver insights by focussing on consistency, efficiency, and efficiency of data samples used for modelling. 


\subsection{Findings from Athey (2015)}

\subsubsection{Key themes explored}

Athey (2015) discusses how machine learning could contribute to causal inference as part of policy evaluations. The discussion is about how studies of policy changes are conducted with applied social science methods, the importance of assessing the robustness of causal estimates, and the problem of inference in studies about large populations.

\subsubsection{Likely changes in economics}

Athey (2015) suggests that the use of machine learning in economic policy evaluation is likely to draw on the strengths of supervised machine learning. The author observes that machine learning approaches have been highly successful in practice when focussed on making predictions, providing data-driven approaches to building models, or validating model selection.

\subsubsection{Aspects of economics that are likely to remain unchanged}

Athey (2015) highlights that the emphasis in economic analysis on ensuring that the same unit (of measurement) is not observed in multiple counterfactual scenarios at the same time will remain unchanged. According to the author, in order to address this 'fundamental problem of causal inference' machine learning methods would need to be modified when dealing with specific behavioural models.

\subsection{Findings from Athey and Luca (2019)}

\subsubsection{Key themes explored}

Athey and Luca (2019) discuss the extent to which PhD economists are increasingly working with technology companies for solving problems related to platform design, pricing, and policy. They discuss how technology companies are hiring not only newly graduated $\mathrm{PhD}$ economists but also economists with tenured positions in well-known university departments.

\subsubsection{Likely changes in economics}

Athey and Luca (2019) suggest that the trend towards increased number of economists being hired in technology companies holds implications for academic papers likely to be published by the economists. They indicate that the emphasis of economists working in technology companies is likely to be on solving business problems. The economists working in technology companies are thus likely to focus on generating specific insights for company strategy and policy. As a result, according to the authors, the research done by these economists is likely to be increasingly about the use of data science/analytics using observational and empirical data to analyse competitors, potential new products, or impact of new initiatives. Increased demand for economists is likely to result in changes to economics curricula with the likely inclusion of programming as a core skill. 


\subsubsection{Aspects of economics that are likely to remain unchanged}

Athey and Luca (2019) indicate that although economists are being hired in technology companies in increased numbers, their work is likely to be mostly in relation to data science or policy teams. Given that the focus of the work economists do in technology companies is likely to be on solving immediate-term business problems, the authors think that it is likely that some of the economists working in technology companies will return to academia to research longterm economic problems. The authors suggest that this may bring a more practice-based perspective to academia and could potentially increase the number of empirical studies in economics.

\subsection{Summary of findings}

\subsubsection{Likely changes in economics}

The articles reviewed in this working paper suggest that the role of machine learning and data science-based approaches in economics is likely to increase in the future. This will likely result in the inclusion of more observational, empirical, data-driven analysis in economics drawing on specific strength of machine learning and data science-based computational methods. Increased visibility and importance of machine learning and data science in economics will likely result in changes to economics curricula at the universities. Increased hiring of economists in technology companies is likely to accelerate the inclusion of practice-based perspective in academic research as economists move between industry and academia.

\subsubsection{Aspects of economics that are likely to remain unchanged}

The existing machine learning and data science literature mainly draws on computer science disciplines. In order to assimilate these approaches successfully in economics further work is needed, for example, to meet the requirements of causal inference, counterfactual analysis, and data sampling. The articles reviewed suggest that although machine learning and data science based approaches will bring changes to economic analysis, the fundamental principles governing economic analysis are unlikely to change. This means machine learning and data science approaches will need to be significantly adapted to meet the prevailing norms of robustness, accuracy, and validation in economics.

\section{References}

Agrawal, Ajay, Joshua Gans, and Avi Goldfarb. 2018a. Prediction Machines: The Simple Economics of Artificial Intelligence. Harvard Business Press.

- 2018b. 'The Economics of Artificial Intelligence'. McKinsey Quarterly.

Athey, Susan. 2015. 'Machine Learning and Causal Inference for Policy Evaluation'. In Proceedings of the 21th ACM SIGKDD International Conference on Knowledge Discovery 
and Data Mining, 5-6. KDD '15. Sydney, NSW, Australia: Association for Computing Machinery. https://doi.org/10.1145/2783258.2785466.

_ 2018. 'The Impact of Machine Learning on Economics'. In The Economics of Artificial Intelligence: An Agenda, 507-547. University of Chicago Press.

Athey, Susan, and Guido W. Imbens. 2019. 'Machine Learning Methods That Economists Should Know About'. Annual Review of Economics 11 (1): 685-725. https://doi.org/10.1146/annurev-economics-080217-053433.

Athey, Susan, and Michael Luca. 2019. 'Economists (and Economics) in Tech Companies'. Journal of Economic Perspectives 33 (1): 209-30. https://doi.org/10.1257/jep.33.1.209.

Ernst, Ekkehardt, Rossana Merola, and Daniel Samaan. 2019. 'Economics of Artificial Intelligence: Implications for the Future of Work'. IZA Journal of Labor Policy 9 (1).

Hsu, Ming-Wei, Stefan Lessmann, Ming-Chien Sung, Tiejun Ma, and Johnnie EV Johnson. 2016. 'Bridging the Divide in Financial Market Forecasting: Machine Learners vs. Financial Economists'. Expert Systems with Applications 61: 215-234.

Kou, Gang, Xiangrui Chao, Yi Peng, Fawaz E Alsaadi, and Enrique Herrera-Viedma. 2019. 'Machine Learning Methods for Systemic Risk Analysis in Financial Sectors'. Technological and Economic Development of Economy 25 (5): 716-742.

Mullainathan, Sendhil, and Jann Spiess. 2017. 'Machine Learning: An Applied Econometric Approach'. Journal of Economic Perspectives 31 (2): 87-106.

VanderPlas, Jake. 2017. Python Data Science Handbook. O'Reilly Media. 